\title{
Liquid crystal behavior of the Kihara fluid
}

\author{
A. Cuetos, B. Martínez-Haya, and S. Lago \\ Departamento de Ciencias Ambientales, Universidad Pablo de Olavide, 41013 Seville, Spain \\ L. F. Rull \\ Departamento de Física Atómica, Molecular y Nuclear, Area de Física Teórica, Universidad de Sevilla, Apartado 1065, \\ 41080 Seville, Spain \\ (Received 28 February 2003; published 15 July 2003)
}

\begin{abstract}
The liquid crystal phases of the Kihara fluid have been studied in computer simulations. The work focuses on the isotropic-nematic-smectic- $A$ triple point region, especially relevant for the understanding of the properties and the design of real mesogens with specific phase diagrams. The Kihara interaction resembles more appropriately than other related models, the shape of elongated polymers and biomolecules, and a closer assertion is provided for the role of the configurational entropy and the dispersive interactions in the behavior of such molecules in dense phases or under macromolecular crowding conditions.
\end{abstract}

DOI: 10.1103/PhysRevE.68.011704

PACS number(s): 64.70.Md, 61.30.Cz, 87.15.Aa

\section{INTRODUCTION}

A number of computationally efficient pair interaction models have been proposed over the past decades for the study of liquid crystalline mesogens. In particular, fluids composed of elongated (prolate) molecules constitute an important class of liquid crystals with relevant industrial and biological applications. Therefore, different models have been used to explore their behavior such as the hard ellipsoid (HE) fluid, the Gay-Berne (GB) fluid, or the hard spherocylinder (HSC) fluid and its square-well (SWSC) or soft repulsive (SRS) variants [1-9].

The Kihara fluid model was introduced in the 1960s [10] as a generalization, for anisotropic molecules, of the spherically symmetric Lennard-Jones fluid [see Eq. (1)] and has been thoroughly employed to investigate the thermodynamic, structural, and transport properties of fluids of linear molecules. In particular, this model has been found to be appropriate to resemble the liquid-vapor equilibrium of different solvents and alkanes of moderate elongation [6]. The Kihara interaction potential incorporates a soft (Lennard-Jones type) attractive well around the molecular core, which is absent or oversimplified in other models investigated previously, such as the SRS or SWSC fluids. The flat square-well attraction of the SWSC fluid, for instance, allows for small compressions or expansions of the system without change of energy. In addition, the spherocylindrical shape of the molecules in the Kihara model is more realistic for many (bio)chemical systems than the ellipsoidal shape imposed by the well-known HE or GB models. For instance, recent investigations have explored the relevance of the spherocylindrical core in the stability of microtubules in solution [11].

We present here a computational study of the Kihara fluid focused on the stability of the isotropic (disordered), nematic (orientational order), and smectic (layering order) liquid crystal phases at different temperatures. To our knowledge, this is the first time that the liquid crystal behavior of this model fluid has been explored, since all of the previous investigations have been restricted to moderate molecular elongations and/or packing fractions corresponding to thermody- namic states deep within the isotropic phase of this system. We hope that the present results will aid in the assessment of the roles of entropy and of the dispersive interactions in the stability of ordered phases of real mesogens and into the appropriate strategy to model them.

\section{METHOD}

We have applied the isothermal-isobaric (NPT) ensemble Monte Carlo (MC) technique to evaluate thermodynamic and structural properties of a Kihara fluid with elongation $L^{*}$ $=L / \sigma=5$. Within this model, the molecules are represented by rigid cylinders of diameter $\sigma$ with semispherical caps (i.e., a so-called spherocylinder) and the pair interaction potential is given by

$$
U(r, \Omega)=\left\{\begin{array}{l}
4 \epsilon\left[\left(\sigma / d_{m}\right)^{12}-\left(\sigma / d_{m}\right)^{6}\right], \quad d_{m} \leqslant 3 \sigma \\
0, \quad d_{m}>3 \sigma
\end{array}\right.
$$

The minimum distance between the central axes of the two prolate molecules, $d_{m}=d_{m}(r, \Omega)$, is a function of their relative orientation $\Omega$, determined by three independent angles, and of the distance between their centers of mass, $r$. For the present simulations, the interaction was truncated at a distance $d_{C}=3 \sigma$, which corresponds to center-of-mass distances ranging from $r=3 \sigma$ to $r=8 \sigma$, depending on the relative pair orientation.

The MC simulations were run over a system of $N_{p}$ $=768$ molecules at four reduced temperatures, $T^{*}=k T / \epsilon$ $=1.5,2,3$, and 5 ( $k$ denotes the Boltzmann constant and $\epsilon$ denotes the well depth) and started from an equilibrated high pressure state well inside the smectic A region of the phase diagram. After equilibration $\left(1 \times 10^{6} \mathrm{MC}\right.$ cycles $)$ and averaging ( $3 \times 10^{5} \mathrm{MC}$ cycles), the last molecular arrangement is stored and used as initial configuration for the subsequent run with a smaller system pressure. Each MC cycle consists of $N_{p}$ attempts for random displacements and/or reorientations of the particles, plus an attempt to change the box volume. The usual periodic boundary conditions are employed and the simulation box is anisotropic with fixed ratios 


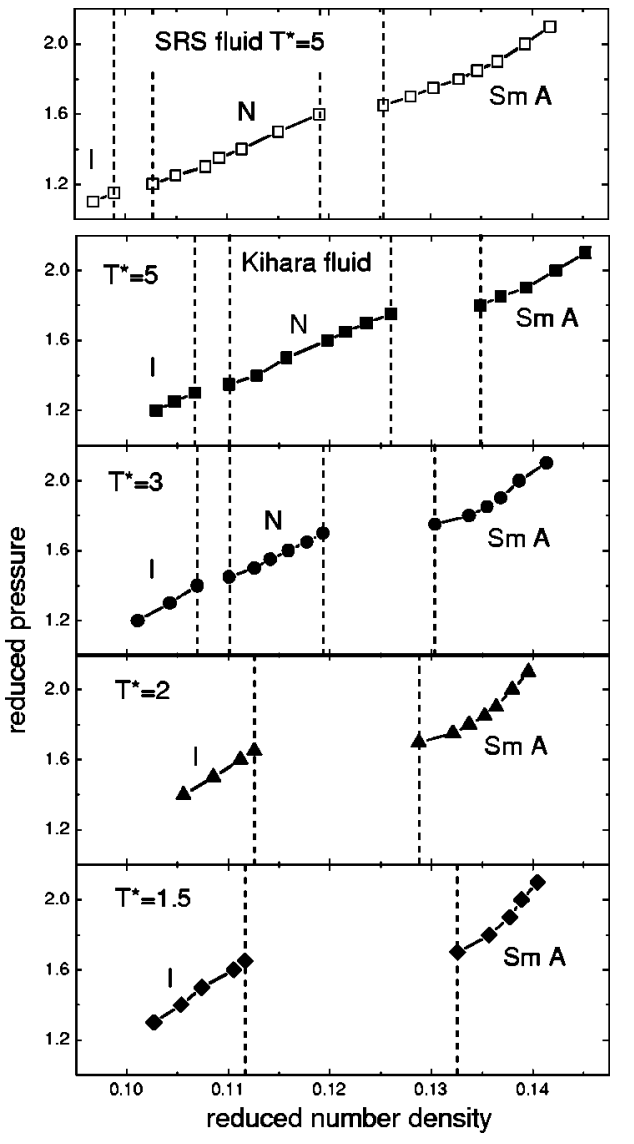

FIG. 1. Equations of state for the isotherms $T^{*}=1.5,2,3$, and 5 of the Kihara fluid with elongation $L^{*}=5$. The vertical lines denote the coexistence densities at the isotropic-nematic $(I-N)$, isotropicsmectic- $A(I-\mathrm{Sm}-A)$ or the nematic-smectic- $A(N-\mathrm{Sm}-A)$ transitions of the fluid. The top panel shows the equation of state of the SRS fluid of same elongation at $T^{*}=5$ (Ref. [5]) for comparison.

$L_{y} / L_{x}=1.15$ and $L_{z} / L_{x}=1.94$. As in our previous study of the SWSC and SRS fluids [5], the longer dimension along the $z$ direction is meant to increase the number of possible smectic layers with respect to the cubic box for a given number of molecules. Further methodological details can be found in Ref. [5]. The (first-order) liquid crystalline transitions observed in the expansion of the fluid are characterized by discontinuities in the density, the nematic order parameter, the bond hexagonal order parameter, and by sudden qualitative changes observed in the different correlation functions $[3,5,12]$. No trace of the hexagonal ordering or long-range correlations characteristic of smectic- $B$ or solidlike phases were found within the scope of the present work.

\section{RESULTS}

The equations of state in reduced units $\left(P^{*}=P \sigma^{3} / k T\right.$ vs $\left.\rho^{*}=\rho \sigma^{3}\right)$ of the $L^{*}=5$ Kihara fluid along the four isotherms investigated are represented in Fig. 1. In addition, Fig. 2 and Table I summarize the coexistence pressures and densities for each of the liquid crystal phase transitions observed.

At the highest temperature investigated, $T^{*}=5$, the $\mathrm{Ki}$ -

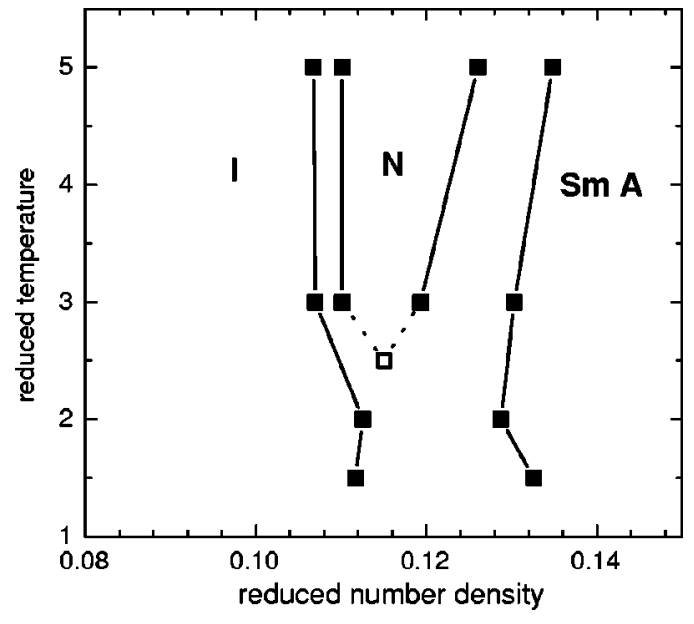

FIG. 2. Summary of the coexistence densities at the isotropicnematic $(I-N)$, isotropic-smectic- $A \quad(I-\mathrm{Sm}-A)$, or nematicsmectic- $A(N-\mathrm{Sm}-A)$ transitions obtained in the present MC-NPT simulations of the Kihara fluid. The open square represents a tentative extrapolation of the results to the $I-N-\mathrm{Sm}-A$ triple point.

hara fluid presents a stable smectic- $A$ phase that undergoes a first-order transition to a nematic phase, as the system is expanded to the pressures below $P^{*}=1.8$. A further expansion of the system induces a transition to an isotropic phase at a pressure just below $P^{*}=1.35$. A qualitatively similar behavior is found in the expansion at $T^{*}=3$. However, at this lower temperature the nematic phase presents a reduced range of stability, as the transition from smectic- $A$ to nematic is delayed to lower pressures and densities in comparison to $T^{*}=5$. Also noticeable is the increased difference in the density of both phases at coexistence. On the other hand, although the subsequent transition to the isotropic phase occurs at a greater reduced pressure in the expansion along the $T^{*}=3$ isotherm than along the $T^{*}=5$ one, the nematicisotropic coexistence densities are very similar at both temperatures.

At the lower temperatures, $T^{*}=1.5$ and $T^{*}=2$, the phase diagram of the Kihara fluid changes qualitatively. The most relevant feature is the absence of the nematic phase and, hence, the appearance of a smectic- $A$-isotropic transition. This kind of phase transition features a greater density asymmetry in the coexistent phases and therefore a stronger firstorder character than the smectic- $A$-nematic or isotropicnematic transitions. Interestingly, the density difference between the smectic- $A$ and isotropic phases at coexistence is greater at $T^{*}=1.5$ than at $T^{*}=2$. This behavior has been also found for the Gay-Berne fluid $[2,12,13]$ and appears to be characteristic of systems with phase diagrams in which the temperature of the $I-N-\mathrm{Sm}-A$ triple point is greater than the critical temperature, which for this fluid is significantly smaller than $T^{*}=1$ [14]. The coexistence states derived from our simulations for the $L^{*}=5$ Kihara fluid allow for an estimation of the triple-point temperature of roughly $T^{*}$ $\approx 2.5$, as tentatively indicated in Fig. 2 .

Figure 1 compares the equations of state and phase transitions at $T^{*}=5$ for the Kihara fluid and its repulsive counterpart, the SRS fluid [5]. The purely repulsive SRS interac- 
TABLE I. Coexistence pressures and densities of the liquid crystal phase transitions for the Kihara fluid and temperatures investigated in this work. The tabulated densities are those of the consecutive states in the MC simulation belonging to different phases, whereas the coexistence pressure is obtained from the mean value of the pressures of the two boundary states. Similar results for the HSC [3], the SWSC, and the SRS fluids [5] are also included for comparison.

\begin{tabular}{|c|c|c|c|c|c|c|c|c|c|}
\hline & \multicolumn{3}{|c|}{$I-\mathrm{Sm}-A$} & \multicolumn{3}{|c|}{$I-N$} & \multicolumn{3}{|c|}{$N-\mathrm{Sm}-A$} \\
\hline & $P^{*}$ & $\rho_{I}^{*}$ & $\rho_{S m A}^{*}$ & $P^{*}$ & $\rho_{I}^{*}$ & $\rho_{N}^{*}$ & $P^{*}$ & $\rho_{N}^{*}$ & $\rho_{S m A}^{*}$ \\
\hline$T^{*}=1.5$ & 1.675 & 0.1117 & 0.1326 & & & & & & \\
\hline$T^{*}=2$ & 1.675 & 0.1126 & 0.1288 & & & & & & \\
\hline$T^{*}=3$ & & & & 1.425 & 0.1069 & 0.1101 & 1.725 & 0.1193 & 0.1294 \\
\hline$T^{*}=5$ & & & & 1.325 & 0.1067 & 0.1101 & 1.775 & 0.1260 & 0.1348 \\
\hline $\operatorname{SRS} T^{*}=5$ & & & & 1.175 & 0.0990 & 0.1027 & 1.625 & 0.1191 & 0.1250 \\
\hline $\operatorname{SWSC} T^{*}=5$ & & & & 1.325 & 0.0968 & 0.0993 & 1.675 & 0.1099 & 0.1155 \\
\hline HSC & & & & 1.190 & 0.0914 & 0.0932 & 1.540 & 0.1061 & 0.1095 \\
\hline
\end{tabular}

tion potential constitutes a generalization of the WeeksChandler-Andersen potential [15] and is built from a shifted Kihara potential truncated at the well minimum. Due to the upward shift of the repulsive wall with respect to the Kihara one, the effective size of the molecules at a given temperature is larger in the SRS model $[4,5]$. Hence, the SRS fluid is expected to be less compressible and to be affected by entropic excluded volume effects to a greater extent than the Kihara fluid, which explains the shift of both the isotropicnematic and the nematic-smectic- $A$ transitions toward lower densities in the SRS system with respect to the Kihara one at $T^{*}=5$ (see Fig. 1). The argumentation is as well consistent with the location of these liquid crystal transitions at even lower densities in the rigid HSC and SWSC fluids [5], as can be seen in Table I.

These latter considerations indicate that, in general terms, the main "driving force" of the isotropic-nematic-smectic transitions in the fluids studied in this work is of entropic nature, the system trying to maximize the volume of phase space accessible to the molecules. The role of the energetic contribution to the free energy is a more subtle, yet relevant one. The most significant effect of the pair interaction potential energy is linked to entropy, since it is the nature of the short-range repulsive interaction that imposes the effective size of the molecules and, thus, the volume excluded by the core of the molecules. Another important qualitative aspect is the role played by the presence of the attractive well in the pair interaction. A first approach to this question is given in our previous work on the SWSC fluid [5], where it was shown that the square-well interaction induces a delay in the $I-N-\mathrm{Sm}-A$ transitions with respect to the purely repulsive HSC fluid (Table I). Thus, the effect of the attractive well affects the transitions in a similar way as a reduction of the effective molecular size would do. Furthermore, the influence of the attractive well should be more relevant at low temperature, but this aspect has not been much explored for fluids of spherocylindrical symmetry [5], even though it is well characterized for the Gay-Berne fluid [2,9,12,13]. A glance at the energetic constrains on the liquid crystal phase transitions as a function of temperature is provided in Fig. 3, which shows the evolution of the average potential energy per particle along the four isotherms of the Kihara fluid studied in this work. Interestingly, the qualitative behavior of the energy at $T^{*}=5$ is quite similar to the one found for the SRS fluid at the same temperature [5] (also shown in Fig. 3 for direct comparison). In both of these cases, the energy grows monotonously with density, except at the nematic-smectic- $A$ transition where a slight decrease in energy is observed. This qualitative trend changes at the lower temperatures, for which a decrease in energy is eventually observed as the density grows within the smectic phase. Hence, it appears that at low temperature the smectic phase is favored by both entropic and energetic constrains. In fact, the increased stabilization of the smectic phase opposes that of the nematic phase, which contributes to the disappearance of this latter phase at $T^{*} \leqslant 2$.

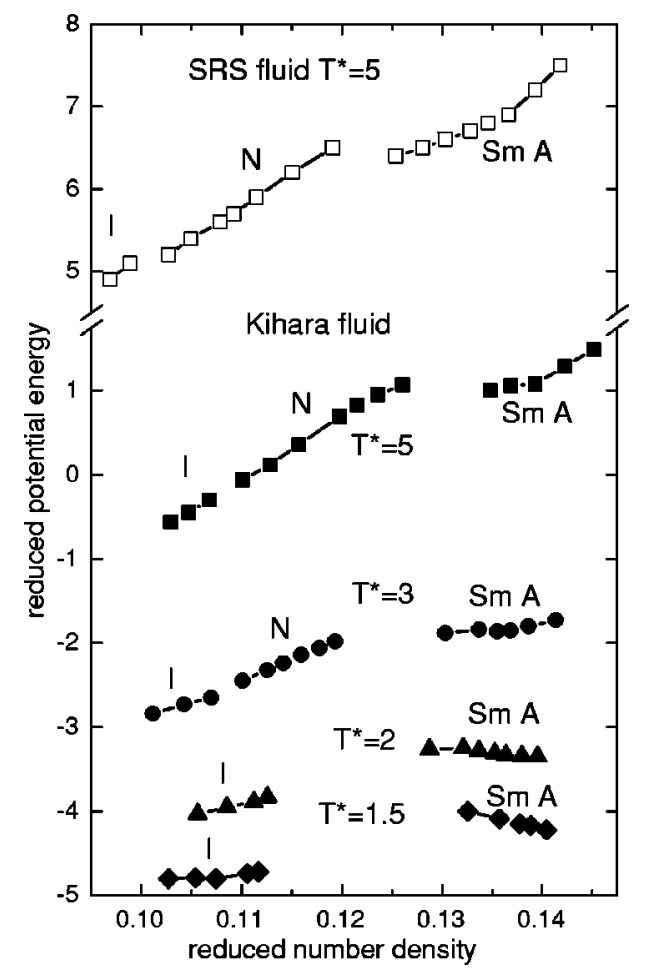

FIG. 3. Pair potential energy along the four isotherms studied in this work for the Kihara fluid. The pair energies for the $T^{*}=5$ isotherm of the SRS fluid from Ref. [5] are also included for comparison. 
A further trend readily noted in Fig. 3 for the Kihara fluid is the global shift of the energy per particle toward more negative values as the temperature is reduced; that is, the attractive contribution to the pair interaction becomes progressively dominant when cooling down the system. The inspection of the radial distribution functions (not shown) indicates that, in fact, the average confinement of neighboring particles within the attractive well is more effective at low temperature, whereas at higher temperatures the pairs show a greater degree of penetration into the short-range repulsive region of the potential.

\section{SUMMARY AND CONCLUSIONS}

It can be concluded that the Kihara potential constitutes a valid model to study the phenomenology of mesogenic fluids. The potential interest of the present results is twofold: they aid to the development of molecular modeling strategies for mesogens with specific phase diagrams and provide a closer assertion of the role played by the configurational entropy and the dispersive interactions in the behavior of molecules in dense environments, typical of liquid crystals but also of the macromolecular crowding conditions of many biological systems.

As already mentioned, the advantage of the Kihara model is that it incorporates a more realistic description of the molecular shape and of the dispersive pair interactions in comparison to other related models. As shown above, the pair interaction in the Kihara fluid brings the liquid crystal phases to significantly higher densities in comparison to the HSC, the SWSC, and the SRS fluids, due to the combined effect of energetic and excluded volume entropic constrains [5]. In particular, the attractive well has a beneficial influence upon the stabilization of the smectic phase with respect to the nematic one and contributes to the destabilization of this latter phase at low temperature.

The present results have served to estimate, for the $L^{*}$ $=5$ fluid, the isotropic-nematic-smectic- $A$ triple point, a thermodynamical parameter relatively scarce in the literature for any of the model fluids commonly employed. A physical interpretation of the supercritical location of the triple point found here for the Kihara fluid can be grasped from the behavior of peptide chains, which are often modelled by rigid long rods. Recent simulations for polyalanine show that the critical temperature of this peptide is well above its decomposition temperature [16]. Since this is a common situation for proteins (with rare exceptions, such as collagen), it may seem that a supercritical triple point, although realistic for ideal stable molecules, would take place at temperatures unattainable for pure systems. However, elongated peptides and, in particular, polyalanine undergo first-order transitions in solution [16]. Our simulations are then relevant within this context, where the effect of solute concentration is analogous to that of an inverse temperature.

The more complete picture of the interplay of entropy and energy in the Kihara fluid would be desirable from an extension of the present work to fluids of varying elongation. In addition, one drawback of the Kihara model is that it assigns the same interaction energy to all pair orientations as long as the minimum distance between the molecules remains constant. In real systems, in contrast, the dispersive interactions are typically greater for aligned configurations than for misaligned configurations of the molecular pairs. This kind of behavior is qualitatively reproduced by models composed of rigid chains with Lennard-Jones interaction sites. Although multiple-site models of this latter type have been employed to study liquid crystal phases $[2,17]$, their use is limited by the computational cost associated to the large number of sites required in order to mimic realistic mesogenic molecules. Our group is at present working on an alternative approach based on a refinement of the Kihara model that incorporates an orientational dependence of the attractive well with the aim to explore the qualitative effects that this feature may introduce in the phase diagram of the fluid.

\section{ACKNOWLEDGMENT}

We acknowledge support from the Spanish Dirección General de Investigación Científica y Técnica (Grant No. BQU2001-3615-C02), Instituto de Salud Carlos III (Grant No. 01/1664), and Plan Andaluz de Investigación (groups FQM-205, FQM-319).
[1] M.P. Allen, G.T. Evans, D. Frenkel, and B.M. Mulder, Adv. Chem. Phys. 86, 1 (1993).

[2] L.F. Rull, Physica A 220, 113 (1995), and citations therein.

[3] S.C. McGrother, D.C. Williamson, and G. Jackson, J. Chem. Phys. 104, 6755 (1996).

[4] D.J. Earl, J. Ilnytskyi, and M. Wilson, Mol. Phys. 99, 1719 (2001).

[5] A. Cuetos, B. Martínez-Haya, L.F. Rull, and S. Lago, J. Chem. Phys. 117, 2934 (2002); 117, 11405 (2002).

[6] S. Lago, B. Garzón, S. Calero, and C. Vega, J. Phys. Chem. 101, 6763 (1997).

[7] M.S. Al-Barwani and M.P. Allen, Phys. Rev. E 62, 6706 (2000).

[8] F. del Río, E. Ávalos, R. Espíndola, L.F. Rull, G. Jackson, and S. Lago, Mol. Phys. 100, 2531 (2002).

[9] M.A. Bates and G.R. Luckhurst, J. Chem. Phys. 110, 7087
(1999).

[10] T. Kihara, Adv. Chem. Phys. 5, 147 (1963).

[11] M. Caplow and L. Fee, Biochemistry 42, 2122 (2003).

[12] J.T. Brown, M.P. Allen, E. Martín del Río, and E. de Miguel, Phys. Rev. E 57, 6685 (1998).

[13] E. de Miguel, E. Martín del Río, J.T. Brown, and M.P. Allen, J. Chem. Phys. 105, 4234 (1996).

[14] B. Martínez-Haya, L.F. Rull, A. Cuetos, and S. Lago, Mol. Phys. 99, 509 (2001).

[15] H.C. Andersen, J.D. Weeks, and D. Chandler, Phys. Rev. A 4, 1597 (1971).

[16] (a) N.A. Alves and U.H.E. Hansmann, Phys. Rev. Lett. 84, 1836 (2000); (b) Y. Peng, U.H.E. Hansmann, and N.A. Alves, J. Chem. Phys. 118, 2374 (2003).

[17] J. Ilnytskyi and M.R. Wilson, Comput. Phys. Commun. 134, 23 (2001). 